\title{
Some Fixed Points Theorems
}

\author{
Vidyadhar V. Nalawade*, Uttam P. Dolhare** \\ *(Department of Mathematics, S. G. R. G. Shinde Mahavidyalaya, Paranda, Dist. Osmanabad, M. S., India \\ 413502, \\ **(Department of Mathematics, D. S. M. College, Jintur, Dist. Parbhani, M. S., India, 431509,
}

\section{ABSTRACT \\ In this research paper we have proved a fixed point theorem by taking a function to be an upper semi-continuous function from right. This theorem uses a function from the set of all positive real numbers to itself. Thus the function appeared out of the metric function as $\psi(d(x, y))$. In the next theorem we have attempted to take the function inside the metric. Precisely, we have defined to be function from a general metric space to itself. Thus in the next theorem it appears like $d(\psi(x), \psi(y))$. We have also presented two examples to prove that the theorems indeed exist. \\ Keywords: Fixed Point, Generalized Contractions, Metric Space, T-Orbitally Complete, Upper Semi- Continuous Function. \\ I. INTRODUCTION \\ Stefan Banach, a celebrated Mathematician from Poland, stated and proved the first astonishing fixed point theorem in 1922, known as the "Banach Contraction Mapping Principle" [1]. This theorem is \\ (M1) $\quad d$ is real-valued, finite and non-negative. \\ (M2) $\quad d(x, y)=0$ if and only if $x=y$, \\ (M3) $\quad d(x, y)=d(y, x)$ \\ (M4) $\quad d(x, y) \leq d(x, z)+d(z, y)$} the origin of Metric Fixed Point Theory. Kannan invented new type of contractions called Kannan Mappings. Kannan proved that his contractions are independent of Banach contractions and also proved that every Kannan mapping on a complete metric space has a unique fixed point [2]. Lj. B. Ćirić in [3] introduced Generalized Contractions and also proved Generalized Contractions include Banach Contractions and Kannan Contractions. Lj. B. Ćirić in the same research paper, proved a fixed point theorem which is a generalization of both Kannan and Banach Fixed Point Theorems. Lj. B. Ćirić diluted the condition of completeness of a metric space to T-Orbitally Completeness. In this research paper we have proved a fixed point theorem by using a somewhat light mode of Generalized Contractions of Lj. B. Ćirić. Our theorem can be illustrated by exhibiting examples from the metric spaces like $\square^{+}$and $l^{2}$. For instance we present an example of the metric space $X=[0,10]$ with the absolute value metric.

\section{PRELIMINARIES AND DEFINITIONS} Definition 2.1 (Metric Space) [4]: A "Metric Space" is a pair $(X, d)$, where $X$ is a set and $d$ is a metric on $X$ (or distance function on $X$ ), that is, a function defined on $X \times X$ such that for all $x, y, z \in X$ we have:
Example 2.1 [4]: The set of all real numbers, taken with the usual metric defined by $d(x, y)=|x-y|$ is a metric space.

Note 2.1 [5]: It is important to note that if $(X, d)$ is a metric space and $A \subseteq X$, then $(A, d)$ is also a metric space.

Definition 2.2: A sequence $\left\{x_{n}\right\}_{n=1}^{\infty}$ in a metric space $(X, d)$ is said to converge or to be convergent if there is an $x \in X$ such that $\lim _{n \rightarrow \infty} d\left(x_{n}, x\right)=0, x$ is called the limit of $\left\{x_{n}\right\}_{n=1}^{\infty}$ and we write $\lim _{n \rightarrow \infty} x_{n}=x$ or $x_{n} \rightarrow x$ as $n \rightarrow \infty$.

Definition 2.3: A sequence $\left\{x_{n}\right\}_{n=1}^{\infty}$ in a metric space $(X, d)$ is said to be a "Cauchy Sequence" is for every $\varepsilon>0$ there is $N=N(\varepsilon)$ such that $d\left(x_{m}, x_{n}\right)<\varepsilon$ for every $m, n \geq N$.

Theorem 2.1: Every convergent sequence in a metric space is a Cauchy sequence.

Note 2.2: The converse of the above theorem is not true in general. That is a Cauchy sequence in a metric space $X$ may or may not converge in $X$. 
Definition 2.4: A metric space $(X, d)$ is said to be a "Complete Metric Space" if every Cauchy Sequence in $X$ converges in $X$.

Definition 2.5: Let $T$ maps a metric space $X$ into $X$. Then the metric space $X$ is said to "T-Orbitally Complete" if every Cauchy sequence of the form $\left\{T^{n} x: x \in X\right\}_{n=1}^{\infty}$ has a limit in $X$.

Definition 2.6 [4]: A "Fixed Point" of a mapping $T: X \rightarrow X$ of a set $X$ into itself is an $x \in X$ which is mapped onto itself, that is $T x=x$.

Definition 2.7 [5]: Let $(X, d)$ be a metric space and let $T$ be a mapping on $X$. Then $T$ is called a "Contraction" if there exists $r \in[0,1)$ such that $d(T x, T y) \leq r d(x, y)$ for all $x, y \in X$.

The following famous theorem is referred to as the Banach Contraction Mapping Principle.

Theorem 2.2 (Banach) [1]: Let $(X, d)$ be a complete metric space and let $T$ be a Contraction on $X$. Then $T$ has a unique fixed point.

Definition 2.8: Le $(X, d)$ be a metric space and let $T$ be a mapping on $X$. Then $T$ is called "Kannan Mapping" if there exists $r \in[0,1 / 2)$ such that $d(T x, T y) \leq r d(x, T x)+r d(y, T y)$ for all $x, y \in X$

Theorem 2.3 (Kannan) [2]: Let $(X, d)$ be a complete metric space and let $T: X \rightarrow X$ be satisfy the condition

$d(T x, T y) \leq r\{d(x, T x)+d(y, T y)\}, \quad$ for $\quad$ all $x, y \in X$, where $r \in\left[0, \frac{1}{2}\right]$, then $T$ has unique fixed point in $X$.

$\mathrm{Lj}$. B. Ćirić introduced the following notion of Generalized Contractions and proved the subsequent theorem.

Definition 2.9 [3]: Let $(X, d)$ be Metric Space and let $T$ be a mapping of $X$ into itself. Then $T$ is said to be a $\lambda$-Generalized Contraction if for every $x, y \in X$ there exists non-negative numbers

$$
\begin{aligned}
& p(x, y), q(x, y), r(x, y), s(x, y) \quad \text { such that } \\
& \operatorname{Sup}_{x, y \in X}\{p(x, y)+q(x, y)+r(x, y)+2 s(x, y)\}=\lambda<1 \\
& \text { and } \\
& d(T x, T y) \leq p d(x, y)+q d(x, T x)+r d(y, T y) \\
& +s\{d(x, T y)+d(y, T x)\}
\end{aligned}
$$

for all $x, y \in X$.
Theorem 2.4 [3]: Let $T$ be $\lambda$-generalized contraction of T-Orbitally Complete metric space $X$ into itself. Then there is a unique point in $u \in X$ which is a fixed point under T, $T^{n} x \rightarrow u$ for every $x \in X, d\left(T^{n} x, u\right) \leq \frac{\lambda^{n}}{1-\lambda} d(x, T x)$.

In the first of our theorems in the next section we use the following definition of an upper semicontinuous function from right.

Definition 2.10: A function $\psi: \square \rightarrow[0, \infty)$ is said to be an "Upper Semi-Continuous from right" if for any sequence $\left\{t_{n}\right\}_{n=1}^{\infty}$ converging to $t \geq 0$, we have $\lim \sup \psi\left(t_{n}\right) \leq \psi(t)$.

\section{MAIN RESULTS}

Theorem 3.1: Let $(X, d)$ be a complete metric space and suppose that $T: X \rightarrow X$ satisfies $d(T x, T y) \leq \alpha \psi(d(x, T x))+\beta \psi(d(y, T y))+\gamma \psi(d(x, y))$ for all $x, y \in X$

Where, $\psi: \square \rightarrow[0, \infty)$ is upper semi continuous from the right and satisfies $0 \leq \psi(t)<t$ for all $t>0, \psi(0)=0$.

Also $0<\alpha+\beta+\gamma<1, \alpha>0, \beta>0, \gamma>0$.

Then $T$ has a unique fixed point in $X$.

Proof: Let $x_{0} \in X$ be an arbitrary but a fixed element in $X$. Define a sequence of iterates $\left\{x_{n}\right\}_{n=1}^{\infty}$ in $X$ by

$x_{1}=T x_{0}, x_{2}=T x_{1}=T^{2} x_{0}, x_{3}=T x_{2}=T^{3} x_{0}, \ldots ., x_{n}=T x_{n-1}=T^{n}$

By the condition (A) on $T$ we get,

$d\left(x_{n}, x_{n+1}\right)=d\left(T x_{n-1}, T x_{n}\right)$

$\leq \alpha \psi\left(d\left(x_{n-1}, T x_{n-1}\right)\right)+\beta \psi\left(d\left(x_{n}, T x_{n}\right)\right)+\gamma \psi\left(d\left(x_{n-1}, x_{n}\right)\right)$

$=\alpha \psi\left(d\left(x_{n-1}, x_{n}\right)\right)+\beta \psi\left(d\left(x_{n}, x_{n+1}\right)\right)+\gamma \psi\left(d\left(x_{n-1}, x_{n}\right)\right)$

$<\alpha d\left(x_{n-1}, x_{n}\right)+\beta d\left(x_{n}, x_{n+1}\right)+\gamma d\left(x_{n-1}, x_{n}\right) \quad(\because \psi(t)<t)$

Thus,

$d\left(x_{n}, x_{n+1}\right)<\alpha d\left(x_{n-1}, x_{n}\right)+\beta d\left(x_{n}, x_{n+1}\right)+\gamma d\left(x_{n-1}, x_{n}\right)$

$\therefore(1-\beta) d\left(x_{n+1}, x_{n}\right)<(\alpha+\gamma) d\left(x_{n-1}, x_{n}\right)$

$\therefore d\left(x_{n+1}, x_{n}\right)<\frac{\alpha+\gamma}{1-\beta} d\left(x_{n-1}, x_{n}\right)$

$\therefore d\left(x_{n+1}, x_{n}\right)<h d\left(x_{n-1}, x_{n}\right)$

Where, $\quad h=\frac{\alpha+\gamma}{1-\beta}$. Here $0<h<1 \quad$ because

$0<\alpha+\beta+\gamma<1, \alpha>0, \beta>0, \gamma>0$. 
Continuing in this way, we get $d\left(x_{n+1}, x_{n}\right)<h^{n} d\left(x_{0}, x_{1}\right)$. Taking limit as $n \rightarrow \infty$ we get,

$d\left(x_{n+1}, x_{n}\right) \rightarrow 0 \quad(\because 0<h<1)$

Therefore

$\left\{x_{n}\right\}_{n=1}^{\infty}$ is a Cauchy sequence in $X$. As $X$ is a complete metric space, there exists $x \in X$ such that $\lim x_{n}=x$. We shall show that $x$ is a fixed point of $T$. As $T$ a is continuous function we have,

$$
x=\lim _{n \rightarrow \infty} x_{n}=\lim _{n \rightarrow \infty} T x_{n-1}=T\left(\lim _{n \rightarrow \infty} x_{n-1}\right)=T x .
$$

Therefore $T x=x$ and $x$ is a fixed point of $T$. Next we shall show that $x$ is unique fixed point of $T$. Let $y \in X$ be another fixed point of $T$.

Again by the condition (A) we get, $d(x, y)=d(T x, T y) \leq \alpha \psi(d(x, T x))+\beta \psi(d(y, T y))+\gamma \psi(d(x, y))$

$$
\begin{aligned}
& =\alpha \psi(d(x, x))+\beta \psi(d(y, y))+\gamma \psi(d(x, y)) \\
& =\alpha \psi(0)+\beta \psi(0)+\gamma \psi(d(x, y)) \\
& =\gamma \psi(d(x, y)) \\
& <\gamma d(x, y)
\end{aligned}
$$

Thus, $d(x, y)<\gamma d(x, y)$

This is possible if and only if $d(x, y)=0$, because $\gamma<1$. Thus $x=y$ and hence the fixed point of $T$ is unique.

Example 3.1: Consider the complete metric space of all non-negative real numbers with absolute value metric. Suppose that $T: X \rightarrow X$ defined by $T x=\frac{x}{8}$. Let, $\quad \psi: \square \rightarrow[0, \infty)$ is defined by $\psi(t)=\frac{t}{2}$. The function $\psi(t)$ is continuous (and hence upper semi-continuous from right), also $0<\psi(t)<t \quad$ for all $t>0, \quad \psi(0)=0$. Let $\alpha=\beta=\gamma=\frac{1}{4}$.

Then clearly

$0<\alpha+\beta+\gamma=\frac{3}{4}<1, \alpha>0, \beta>0, \gamma>0$.

We verify that the condition (A) of the theorem 4.1 is satisfied. We observe that $d(T x, T y)=d\left(\frac{x}{8}, \frac{y}{8}\right)=\frac{|x-y|}{8}$.

Also

$$
\begin{aligned}
& \alpha d(x, T x)+\beta d(y, T y)+\gamma d(x, y) \\
& =\frac{1}{4} \psi\left(d\left(x, \frac{x}{8}\right)\right)+\frac{1}{4} \psi\left(d\left(y, \frac{y}{8}\right)\right)+\frac{1}{4} \psi(d(x, y)) \\
& =\frac{1}{4} \psi\left(\frac{7 x}{8}\right)+\frac{1}{4} \psi\left(\frac{7 y}{8}\right)+\frac{1}{4} \psi(|x-y|) \\
& =\frac{1}{4} \frac{\left(\frac{7 x}{8}\right)}{2}+\frac{1}{4} \frac{\left(\frac{7 y}{8}\right)}{2}+\frac{1}{4} \frac{|x-y|}{2}=\frac{7 x}{64}+\frac{7 y}{64}+\frac{|x-y|}{8} \\
& =\frac{7(x+y)}{64}+\frac{|x-y|}{8} .
\end{aligned}
$$

Thus clearly

$$
\begin{aligned}
& d(T x, T y)=d\left(\frac{x}{8}, \frac{y}{8}\right)=\frac{|x-y|}{8} \\
& <\frac{7(x+y)}{64}+\frac{|x-y|}{8} \\
& =\alpha d(x, T x)+\beta d(y, T y)+\gamma d(x, y)
\end{aligned}
$$

for all $x \in \square^{+}$. The condition (A) of the theorem 4.1 is satisfied. We see that $x=0$ is the unique fixed point of the function $T$.

Theorem 3.2: Let $(X, d)$ be a T-Orbitally Complete metric space with respect to a mapping $T: X \rightarrow X$. Let $*$ be the operation defined on the set $X$ by $\alpha * x$ for $\alpha \in \square$ and $x \in X$, such that $\alpha * x \in X$. Also let the metric $d$ satisfies $d(\alpha x, \alpha y)=|\alpha| d(x, y)$ for any scalar $\alpha \in \square$. Further let there exist a number $k \in \square^{+}$such that,

(B) $\operatorname{Sup}_{x, y \in X}(p(x, y)+q(x, y)+r(x, y)+2 s(x, y))=\lambda<\frac{1}{k}$ for some non-negative numbers $p(x, y), q(x, y), r(x, y), s(x, y)$, which may depend on $x$ and $y$,

(C) For the function $\psi: X \rightarrow X$ defined by $\psi(x)=k x, k \in \square, T$ satisfies the condition $d(T x, T y) \leq$

$p d(\psi(x), \psi(y))+q d(\psi(x), \psi(T x))+$

$r d(\psi(y), \psi(T y))+$

$s\{d(\psi(x), \psi(T y))+d(\psi(y), \psi(T x))\}$

for all $x, y \in X$.

Then $T$ has a unique fixed point in $X$.

Proof: For the sake of simplicity we shall denote $\alpha * x$ by $\alpha x$. Let $x_{0} \in X \quad x_{0} \in X$ be arbitrary and define a sequence,

$x_{1}=T x_{0}, x_{2}=T x_{1}, x_{3}=T x_{2}, \ldots . ., x_{n+1}=T x_{n}, \ldots \ldots$

Now by using the condition (C) in the statement of 
the theorem and triangle inequality, we get,

$d\left(x_{n}, x_{n+1}\right)$

$=d\left(T x_{n-1}, T x_{n}\right)$

$\leq p d\left(\psi\left(x_{n-1}\right), \psi\left(x_{n}\right)\right)+q d\left(\psi\left(x_{n-1}\right), \psi\left(T x_{n-1}\right)\right)+$

$r d\left(\psi\left(x_{n}\right), \psi\left(T x_{n}\right)\right)+s\left\{d\left(\psi\left(x_{n-1}\right), \psi\left(T x_{n}\right)\right)+d\left(\psi\left(x_{n}\right), \psi\left(T x_{n-1}\right)\right)\right\}$

$=p d\left(\psi\left(x_{n-1}\right), \psi\left(x_{n}\right)\right)+q d\left(\psi\left(x_{n-1}\right), \psi\left(x_{n}\right)\right)+$

$r d\left(\psi\left(x_{n}\right), \psi\left(x_{n+1}\right)\right)+s\left\{d\left(\psi\left(x_{n-1}\right), \psi\left(x_{n+1}\right)\right)+d\left(\psi\left(x_{n}\right), \psi\left(x_{n}\right)\right)\right\}$

$=p d\left(k x_{n-1}, k x_{n}\right)+q d\left(k x_{n-1}, k x_{n}\right)+$

$r d\left(k x_{n}, k x_{n+1}\right)+s\left\{d\left(k x_{n-1}, k x_{n+1}\right)+d\left(k x_{n}, k x_{n}\right)\right\}$

$=(p k) d\left(x_{n-1}, x_{n}\right)+(q k) d\left(x_{n-1}, x_{n}\right)+$

$(r k) d\left(x_{n}, x_{n+1}\right)+(s k)\left\{d\left(x_{n-1}, x_{n+1}\right)+d\left(x_{n}, x_{n}\right)\right\}$

$=(p k) d\left(x_{n-1}, x_{n}\right)+(q k) d\left(x_{n-1}, x_{n}\right)+$

(rk)d $\left(x_{n}, x_{n+1}\right)+(s k) d\left(x_{n-1}, x_{n+1}\right)$

$\leq(p k) d\left(x_{n-1}, x_{n}\right)+(q k) d\left(x_{n-1}, x_{n}\right)+$

$(r k) d\left(x_{n}, x_{n+1}\right)+(s k)\left\{d\left(x_{n-1}, x_{n}\right)+d\left(x_{n}, x_{n+1}\right)\right\}$

Thus we have,

$d\left(x_{n}, x_{n+1}\right) \leq(p k) d\left(x_{n-1}, x_{n}\right)+(q k) d\left(x_{n-1}, x_{n}\right)+$

$$
(r k) d\left(x_{n}, x_{n+1}\right)+(s k)\left\{d\left(x_{n-1}, x_{n}\right)+d\left(x_{n}, x_{n+1}\right)\right\}
$$

$\Rightarrow[1-r k-s k] d\left(x_{n}, x_{n+1}\right) \leq[p k+q k+s k] d\left(x_{n-1}, x_{n}\right)$

$\Rightarrow d\left(x_{n}, x_{n+1}\right) \leq \frac{p k+q k+s k}{1-r k-s k} d\left(x_{n-1}, x_{n}\right)$

$\Rightarrow d\left(x_{n}, x_{n+1}\right) \leq \frac{p+q+s}{\left(\frac{1}{k}\right)-r-s} d\left(x_{n-1}, x_{n}\right)$

$\Rightarrow d\left(x_{n}, x_{n+1}\right) \leq h d\left(x_{n-1}, x_{n}\right)$

Here $h=\frac{p+q+s}{\left(\frac{1}{k}\right)-r-s}<1$ (by condition (1) in the

theorem).

Repeating this argument, ultimately we reach to the step

$d\left(x_{n}, x_{n+1}\right) \leq h^{n} d\left(x_{0}, x_{1}\right)$

When we take the limit as $n \rightarrow \infty$ we get $h \rightarrow 0$ as $h<1$. Thus it follows from (1) that $\left\{x_{n}\right\}_{n=1}^{\infty}$ is a Cauchy Sequence. Since $X$ is T-Orbitally Complete, there is a point $x \in X$, such that $x=\lim _{n \rightarrow \infty} T^{n} x_{0}=\lim _{n \rightarrow \infty} x_{n}$

We shall show that $x$ is a fixed point of $T$.

Using the conditions (B) and (C) in the statement of the theorem and triangle inequality we get,
$d\left(T x, T x_{n}\right)$

$\leq p d\left(\psi(x), \psi\left(x_{n}\right)\right)+q d(\psi(x), \psi(T x))+$ $r d\left(\psi\left(x_{n}\right), \psi\left(T x_{n}\right)\right)+s\left\{d\left(\psi(x), \psi\left(T x_{n}\right)\right)+d\left(\psi\left(x_{n}\right), \psi(T x)\right)\right\}$

$=p d\left(\psi(x), \psi\left(x_{n}\right)\right)+q d(\psi(x), \psi(T x))+$ $r d\left(\psi\left(x_{n}\right), \psi\left(x_{n+1}\right)\right)+s\left\{d\left(\psi(x), \psi\left(x_{n+1}\right)\right)+d\left(\psi\left(x_{n}\right), \psi(T x)\right)\right\}$

$=p d\left(k x, k x_{n}\right)+q d(k x, k T x)+r d\left(k x_{n}, k x_{n+1}\right)+$ $s\left\{d\left(k x, k x_{n+1}\right)+d\left(k x_{n}, k T x\right)\right\}$ $=(p k) d\left(x, x_{n}\right)+(q k) d(x, T x)+(r k) d\left(x_{n}, x_{n+1}\right)+$ (sk) $\left\{d\left(x, x_{n+1}\right)+d\left(x_{n}, T x\right)\right\}$ $\leq(p k) d\left(x, x_{n}\right)+(q k)\left\{d\left(x, x_{n+1}\right)+d\left(x_{n+1}, T x\right)\right\}+$ $(r k) d\left(x_{n}, x_{n+1}\right)+(s k)\left\{d\left(x, x_{n+1}\right)+d\left(x_{n}, T x\right)\right\}$

$=(p k) d\left(x, x_{n}\right)+[q k+s k] d\left(x, x_{n+1}\right)+$

$(q k) d\left(T x_{n}, T x\right)+(r k) d\left(x_{n}, x_{n+1}\right)+(s k) d\left(x_{n}, T x\right)$

$($ Triangle Inequátilipk $) d\left(x, x_{n}\right)+[q k+s k] d\left(x, x_{n+1}\right)+(q k) d\left(T x_{n}, T x\right)+$

$(r k) d\left(x_{n}, x_{n+1}\right)+(s k)\left\{d\left(T x, T x_{n}\right)+d\left(T x_{n}, x_{n}\right)\right\}$

$\leq(p k) d\left(x, x_{n}\right)+[q k+s k] d\left(x, x_{n+1}\right)+$

$[q k+s k] d\left(T x_{n}, T x\right)+[r k+s k] d\left(x_{n}, x_{n+1}\right)$

$\leq \lambda d\left(x, x_{n}\right)+\lambda d\left(x, x_{n+1}\right)+\lambda d\left(T x_{n}, T x\right)+\lambda d\left(x_{n}, x_{n+1}\right)$

$=\lambda\left\{d\left(x, x_{n}\right)+d\left(x, x_{n+1}\right)+d\left(x_{n}, x_{n+1}\right)\right\}+\lambda d\left(T x_{n}, T x\right)$

Consequently we have

$d\left(T x, T x_{n}\right) \leq \lambda\left\{\begin{array}{l}d\left(x, x_{n}\right)+d\left(x, x_{n+1}\right)+ \\ d\left(x_{n}, x_{n+1}\right)\end{array}\right\}+\lambda d\left(T x_{n}, T x\right)$

$\Rightarrow[1-\lambda] d\left(T x, T x_{n}\right) \leq \lambda\left\{\begin{array}{l}d\left(x, x_{n}\right)+ \\ d\left(x, x_{n+1}\right)+d\left(x_{n}, x_{n+1}\right)\end{array}\right\}$

$\Rightarrow d\left(T x, T x_{n}\right) \leq \frac{\lambda}{1-\lambda}\left\{\begin{array}{l}d\left(x, x_{n}\right)+ \\ d\left(x, x_{n+1}\right)+d\left(x_{n}, x_{n+1}\right)\end{array}\right\}$.

Taking limit as $n \rightarrow \infty$ we get

$\lim _{n \rightarrow \infty} d\left(T x, T x_{n}\right) \leq \frac{\lambda}{1-\lambda} \lim _{n \rightarrow \infty}\left\{\begin{array}{l}d\left(x, x_{n}\right)+d\left(x, x_{n+1}\right)+ \\ d\left(x_{n}, x_{n+1}\right)\end{array}\right\}$ $=0$

(by (2) and the fact that $\left\{x_{n}\right\}_{n=1}^{\infty}$ is a Cauchy Sequence).

Thus we get $T x=\lim _{n \rightarrow \infty} T x_{n}=\lim _{n \rightarrow \infty} x_{n+1}=x$.

We have shown that $T$ has a fixed point $x \in X$. Uniqueness: Let there is $y \in X$ such that $T y=y$. Again using the conditions (B) and $(\mathrm{C})$ we get, 
$d(x, y)$

$=d(T x, T y)$

$\leq p d(\psi(x), \psi(y))+q d(\psi(x), \psi(T x))+$

$r d(\psi(y), \psi(T y))+s\{d(\psi(x), \psi(T y))+d(\psi(y), \psi(T x))\}$

$=p d(\psi(x), \psi(y))+q d(\psi(x), \psi(x))+$

$r d(\psi(y), \psi(y))+s\{d(\psi(x), \psi(y))+d(\psi(y), \psi(x))\}$

$=p d(k x, k y)+s\{d(k x, k y)+d(k y, k x)\}$

$=(p k) d(x, y)+2(s k) d(x, y)$

$=[p k+2(s k)] d(x, y)$

$\leq \lambda d(x, y)$

Thus we have $d(x, y) \leq \lambda d(x, y)$. This implies that $[1-\lambda] d(x, y) \leq 0$. That is $x=y$. Hence the fixed point of $T$ is unique.

Example 3.2: Consider the metric space $X=[0,10]$ with the usual metric $d$ of absolute value. That is $d(x, y)=|x-y|$ for all $x, y \in X$.

$d$ satisfies

$d(\alpha x, \alpha y)=|\alpha x-\alpha y|=|\alpha||x-y|=|\alpha| d(x, y)$ for all $x, y \in X$. Define $T: X \rightarrow X$ by $T x=\frac{3}{4} x$ for all $x \in X$. Then $X$ is T-Orbitally Complete metric space. Then for $k=\frac{1}{2}$, we observe that

1) There exists numbers

$$
\begin{aligned}
& p(x, y)=\frac{3}{2}, q(x, y)=\frac{1}{10} \\
& r(x, y)=\frac{1}{10}, s(x, y)=\frac{1}{10}
\end{aligned}
$$

such that

$$
\begin{gathered}
\operatorname{Sup}_{x, y \in X=[0,10]}\{p+q+r+2 s\}=\operatorname{Sup}_{x, y \in X=[0,10]}\left\{\frac{3}{2}+\frac{1}{10}+\frac{1}{10}+2 \frac{1}{10}\right\}= \\
\frac{19}{10}=1.9<2=\frac{1}{\left(\frac{1}{2}\right)}=\frac{1}{k} .
\end{gathered}
$$

2) For the function $\psi: X \rightarrow X$ defined by

$$
\psi(x)=k x=\frac{1}{2} x \text { for all } x \in X \text {, we have }
$$$$
d(T x, T y)=d\left(\frac{3}{4} x, \frac{3}{4} y\right)=\left|\frac{3}{4} x-\frac{3}{4} y\right|=\frac{3}{4}|x-y| .
$$

And

$$
\begin{aligned}
& p d(\psi(x), \psi(y))+q d(\psi(x), \psi(T x))+r d(\psi(y), \psi(T y))+ \\
s & \{d(\psi(x), \psi(T y))+d(\psi(y), \psi(T x))\} \\
= & \frac{3}{2} d(\psi(x), \psi(y))+\frac{1}{10} d\left(\psi(x), \psi\left(\frac{3}{4} x\right)\right)+\frac{1}{10} d\left(\psi(y), \psi\left(\frac{3}{4} y\right)\right)+ \\
& \frac{1}{10}\left\{d\left(\psi(x), \psi\left(\frac{3}{4} y\right)\right)+d\left(\psi(y), \psi\left(\frac{3}{4} x\right)\right)\right\} \\
= & \frac{3}{2} d\left(\frac{1}{2} x, \frac{1}{2} y\right)+\frac{1}{10} d\left(\frac{1}{2} x, \frac{1}{2} \frac{3}{4} x\right)+\frac{1}{10} d\left(\frac{1}{2} y, \frac{1}{2} \frac{3}{4} y\right)+ \\
& \frac{1}{10}\left\{d\left(\frac{1}{2} x, \frac{1}{2} \frac{3}{4} y\right)+d\left(\frac{1}{2} y, \frac{1}{2} \frac{3}{4} x\right)\right\} \\
= & \frac{3}{2} \frac{1}{2} d(x, y)+\frac{1}{10} \frac{1}{2} d\left(x, \frac{3}{4} x\right)+\frac{1}{10} \frac{1}{2} d\left(y, \frac{3}{4} y\right)+ \\
& \frac{1}{10} \frac{1}{2}\left\{d\left(x, \frac{3}{4} y\right)+d\left(y, \frac{3}{4} x\right)\right\} \\
= & \frac{3}{4}|x-y|+\frac{1}{20}\left(\frac{1}{4} x\right)+\frac{1}{20}\left(\frac{1}{4} y\right)+\frac{1}{20}\left\{\frac{|4 x-3 y|+|4 y-3 x|}{4}\right\}
\end{aligned}
$$

Thus

clearly

$$
\begin{aligned}
d(T x, T y) \leq & p d(\psi(x), \psi(y))+q d(\psi(x), \psi(T x))+ \\
& r d(\psi(y), \psi(T y))+s\left\{\begin{array}{l}
d(\psi(x), \psi(T y))+ \\
d(\psi(y), \psi(T x))
\end{array}\right\}
\end{aligned}
$$

for all $x, y \in X$. The conditions (B) and (C) of the theorem 4.2 are satisfied. We see that $x=0$ is the unique fixed point of $T$ in $X$.

Remark 4.1: In the theorem 4.2, if we take $k=1$ we get the Lj. B. Ćirić theorem 3.4.

\section{CONCLUSION}

The advantage of the theorem 3.2 is that the function $\psi$ may be taken inside the distance function. This may open new possibilities of studies in fixed point theory. These theorems are applicable to attempt solutions to differential equations, economics and game theory.

\section{ACKNOWLEDGEMENTS}

Authors are thankful to the referees for their valuable suggestions.

\section{REFERENCES}

[1] S. Banach, Sur les opérations dans les ensembles abstraits et leur applications aux equations intégrales. Fund. Math. 3, 133181 (1922).

[2] R. Kannan, Some results on fixed points II, Amer. Math. Monthly, 76 (1969), 405408, MR0257838 (41:2487).

[3] Lj. B. Ćirić, Generalized Contractions and fixed-point theorems, Publications Inst. Math., Nouv. série, tome 12 (26), 1971, 1926. 
[4] Kreyszig, E., Introductory Functional Analysis with Applications, (Wiley Classics Library. Wiley, New York, 1989).

[5] Richard R. Goldberg, Methods of Real Analysis, (Oxford and IBH Publishing Co. Pvt. Ltd., New Delhi, 1970).

International Journal of Engineering Research and Applications (IJERA) is UGC approved Journal with S1. No. 4525, Journal no. 47088.

Vidyadhar V. Nalawade. "Some Fixed Points Theorems." International Journal of Engineering Research and Applications (IJERA) 7.7 (2017): 51-56. 